\title{
Article
}

\section{Quantification and confocal imaging of protein specific molecularly imprinted polymers}

Hawkins, DM, Trache, A, Ellis, EA, Stevenson, D, Holzenburg, A, Meininger, GA and Reddy, Subrayal M

Available at https://clok.uclan.ac.uk/13680/

Hawkins, DM, Trache, A, Ellis, EA, Stevenson, D, Holzenburg, A, Meininger, GA and Reddy, Subrayal M orcid iconORCID: 0000-0002-7362-184X (2006) Quantification and confocal imaging of protein specific molecularly imprinted polymers. Biomacromolecules, 7 (9). 2560 - 2564. ISSN 1525-7797

It is advisable to refer to the publisher's version if you intend to cite from the work. http://dx.doi.org/10.1021/bm060494d

For more information about UCLan's research in this area go to http://www.uclan.ac.uk/researchgroups/ and search for <name of research Group>.

For information about Research generally at UCLan please go to http://www.uclan.ac.uk/research/

All outputs in CLoK are protected by Intellectual Property Rights law, including Copyright law. Copyright, IPR and Moral Rights for the works on this site are retained by the individual authors and/or other copyright owners. Terms and conditions for use of this material are defined in the policies page.

\section{CLoK}

Central Lancashire online Knowledge www.clok.uclan.ac.uk

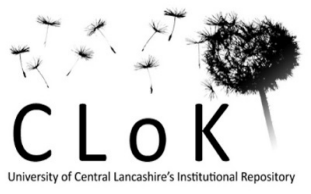




\title{
Quantification and Confocal Imaging of Protein
}

\section{Specific Molecularly Imprinted Polymers}

\section{(HydroMIPs)}

\author{
Daniel M. Hawkins* ${ }^{\star}$ Andreea Trache ${ }^{\uparrow}$, E. Ann Ellis, \\ Derek Stevenson*, Andreas Holzenburg ${ }^{\ddagger}$, Gerald A. Meininger ${ }^{\uparrow}$, \\ \& Subrayal M. Reddy*
}

Email: d.m.hawkins@surrey.ac.uk; trache@tamu.edu; ellisa@mic.tamu.edu; d.stevenson@surrey.ac.uk; holzen@mic.tamu.edu; gam@tamu.edu; s.reddy@surrey.ac.uk

${ }^{*}$ School of Biomedical and Molecular Sciences, University of Surrey, Guildford, Surrey, GU2 7XH, UK;

${ }^{\uparrow}$ Department of Systems Biology and Translational Medicine, College of Medicine, Reynolds Medical Building, Texas A\&M University-Health Science Centre, College Station, TX 77843-1114, USA.

¥Microscopy \& Imaging Centre, Texas A\&M University, College Station, TX 77843, USA.

Corresponding Author: Tel: +44 (0)148368 6396, Fax: +44 (0)1483686401 e-mail: s.reddy@surrey.ac.uk (S.M.Reddy) 


\section{Abstract}

We have employed FITC-albumin as the protein template molecule in an aqueous phase molecular imprinted polymer (HydroMIP) strategy. For the first time, the use of a fluorescently labelled template is reported, with subsequent characterisation of the smart material to show that the HydroMIP possess a significant molecular memory in comparison to that of the nonimprinted control polymer (HydroNIP). The imaging of the FITC-albumin imprinted HydroMIP using confocal microscopy is described, with the in situ removal of imprinted protein displayed in terms of observed changes in the fluorescence of the imprinted polymer, both before and after template elution (using a $10 \% \mathrm{SDS} / 10 \% \mathrm{AcOH}(\mathrm{w} / \mathrm{v}$ ) solution). We also report the imaging of a bovine haemoglobin (BHb) imprinted HydroMIP using two-photon confocal microscopy, and describe the effects of template elution upon protein autofluorescence. The findings further contribute to the understanding of aqueous phase molecular imprinting protocols, and document the use of fluorescence as a useful tool in template labelling/detection and novel imaging strategies.

\section{Keywords}

Molecularly Imprinted Polymers, HydroMIPs, Proteins, Fluorescent Imaging, Autofluorescence, Biosensors, Protein Markers. 


\section{Introduction}

The continual need for novel, inexpensive, fast and efficient diagnostic methods within the pharmaceutical, clinical and other sectors have fuelled much research into the development of highly sensitive analytical procedures. In some methodologies selective and sensitive procedures require sophisticated and expensive equipment, as well as skilled operatives. An alternative approach is the development of highly selective capture reagents such as antibodies or artificial antibody mimics, such as molecularly imprinted polymers (MIPs). MIPs have become an important tool in the preparation of artificial and robust recognition materials that are capable of mimicking natural systems. When compared to molecular recognition products such as antibodies, MIPs bring advantages such as durability, specificity and ease of mass production, that have previously not been offered by alternative techniques.

The principle of Molecular Imprinting (MI) relies on the formation of a host-guest complex between a template molecule of interest, and one or more functional monomers. The template is allowed to associate with the functional monomers in an appropriate solvent, and a plethora of non-covalent interactions are formed. Upon the addition of a crosslinking agent and subsequent polymerisation, a rigid, highly crosslinked polymer matrix is formed which encapsulates the template molecule within. After removing the template molecule, an "imprint" remains, which is complimentary to the original molecule in terms of shape, size and distribution of charge.

Despite the promise that MI holds as an analytical technique, its application has been largely reserved for the imprinting of low molecular 
weight molecules, and has failed to compete with the more established immunological based techniques that are currently widely employed for the detection of macromolecular templates, especially proteins. This has occurred primarily due to the reliance upon organic solvents to promote the formation of the host-guest interactions that govern the technique. As a direct result, the imprinting of molecules of biological significance has proven to be particularly problematic, and consequently the progression of the technique into a clinical or commercial setting has been hampered.

Progression and development of the use of MIPs as an analytical device depends on the successful synthesis of chemically and mechanically stable MIPs in aqueous media that demonstrate the ability to specifically and selectively recognise biological molecules. Polyacrylamide hydrogels are beginning to gain popularity as suitable aqueous based matrices for MIPs, and the optimisation of imprinting protocols for a number of biological molecules of significance has been successfully achieved $(1,2,3)$. These aqueous phase MIPs or 'HydroMIPs' hold much promise for the future development of novel sensing strategies for a whole host of biological molecules. Most studies on MIPs concentrate on selective binding and rebinding of the template molecule. Few studies directly demonstrate the architecture of the cavities formed. Studies such as these reported here should help explain some of the unexpected selectivity previously reported for $\operatorname{MIPs}(4)$.

In recent decades, the emergence of fluorescent proteins has initiated a revolution in the study of biological processes ranging from gene expression at the cellular level to systems physiology. However, it is the recent technical 
advances in quantitative imaging of these fluorescent bodies using confocal microscopy, the bona fide gold standard imaging technique in biology, that has truly advanced our knowledge. MI may potentially play a key role in the development of detection techniques for fluorescent or fluorescently labelled molecules, and the robust ability to engineer HydroMIPs should enable the rapid progression and commercial development of diagnostic tests based upon the utilisation of HydroMIPs as the selective recognition element of a biosensor strategy.

Many modern imaging techniques, although extremely powerful in nature, require a great deal of sample preparation, which is necessary to enable analysis under high vacuums. Confocal microscopy requires little sample preparation and can be operated in ambient surroundings without the constraints of specific experimental parameters. Therefore, the structure of the polyacrylamide hydrogels, as well as biological template molecules that may be embedded within the polymer matrix can potentially be visualised in their native form, and considerations regarding the effects of sample preparation upon the degradation of sample structure are minimised. Additionally, the capability of being able to image both fluorescent and autofluorescent molecules, combined with the technical advantages such as imaging with reduced out of focus light, controllable depth of field and real time image acquisition, make confocal microscopy a highly powerful analytical tool.

To date, little work has been conducted regarding the molecular imprinting of fluorescent molecules, primarily due to the inherent disadvantages of the technique as discussed. Fluorescent functional 
monomers have been investigated for the imprinting of a variety of small molecules, where fluorescence intensity alters upon a specific binding event or template-cavity interaction(5-9). Fluorescent detection of small organic template molecules has been extensively employed in different forms ${ }^{(10,11)}$, and hydrogels have been imaged using confocal microscopy and employed in a variety of differing functional roles ${ }^{(12-14)}$. Additionally, fluorescently labelled hydrogels have also been imaged using confocal microscopy, with the swelling characteristics, quenching effects and thermo-responsive nature of polyacrylamide gels being of particular interest $(15,16)$

Albumin is a large and robust protein molecule suitable for aqueous phase molecular imprinting as it is a clinically significant biomarker of a variety of pathophysiological conditions, and detection of such a molecule would further develop the understanding of HydroMIPs as novel recognition materials. Fluorescein is a fluorophore commonly used in microscopy, with the reactive fluorescein isothiocyanate derivative (FITC) used to label and track biological molecules in various microscopy applications. Conjugation of FITC to proteins such as albumin occurs via primary amines (lysines) and results in between 8-12 FITC conjugates to each protein molecule.

Up to now, confocal microscopy has not been employed to image the presence of fluorescent template molecules within aqueous phase molecularly imprinted polymers. We report of the design and manufacture of a polyacrylamide based HydroMIP for an FITC labelled albumin template molecule and detail its structure using confocal microscopy. We also report the confocal analysis of a bovine haemoglobin (BHb) specific HydroMIP, and 
detail the effects of in situ, elution of the template molecule from the HydroMIP matrix.

\section{Experimental Section}

\section{HydroMIP production}

BHb (12mg), acrylamide (54mg), N,N'-methylenebisacrylamide (6mg) and $10 \mu \mathrm{l}$ of $10 \%(\mathrm{w} / \mathrm{v})$ ammonium persulfate (APS) were dissolved in $1 \mathrm{ml}$ of reverse osmosis $(\mathrm{RO})$ water in a $7 \mathrm{ml}$ polystyrene/polyethylene bijou. $20 \mu \mathrm{l}$ of $5 \%(\mathrm{v} / \mathrm{v}) \mathrm{N}, \mathrm{N}, \mathrm{N}^{\prime}, \mathrm{N}^{\prime}$-tetramethylethyldiamine was added and the solution was deoxygenated by purging with nitrogen for 5 mins. Polymerisation occurred overnight at room temperature to give a polymer hydrogel that possessed a final cross-linking concentration of $10 \%$ (quantified in terms of the amount of cross-linker in relation to the total monomer composition of the gel). FITCalbumin gels were also prepared in an identical manner (using 6mg of protein) and non-imprinted control gels were made in the absence of both $\mathrm{BHb}$ and FITC-albumin. Characterisation of the "imprinting effect" exhibited by the $\mathrm{BHb}$ specific HydroMIPs has previously been described by the authors(3).

\section{Characterisation of FITC-albumin "Imprinting Effect"}

FITC-albumin gels were granulated by passing through a $75 \mu \mathrm{m}$ sieve (Endecotts Ltd, London, UK), transferred to $10 \mathrm{ml}$ polystyrene/polyethylene centrifuge tubes and washed with five $2 \mathrm{ml}$ volumes of $\mathrm{RO}$ water followed by five $2 \mathrm{ml}$ volumes of a $10 \%(\mathrm{w} / \mathrm{v})$ sodium dodecyl sulphate/acetic acid (SDS:AcOH) eluant (purchased from Sigma (Poole, UK) and Fisher Scientific (Loughborough, UK) respectively). Each wash/elution step was performed by centrifugation at 3000 rpm for 5 mins using a Centaur II centrifuge (Fisher 
Scientific, Loughborough, UK), with all supernatant fractions extracted by pipette and collected for analysis.

Calibration curves $(0-0.3 \mathrm{mg} / \mathrm{ml})$ were prepared for FITC-albumin, in the respective wash and elution solvents used, allowing the accurate measurement of template removal under the corresponding acid/surfactant conditions. The FITC-albumin concentration of the calibration standards and all experimentally derived wash/elution fractions was determined by pipetting $200 \mu \mathrm{l}$ of each solution into white 96 well plates (Fisher Scientific, Loughborough, UK), and analysing on a PerkinElmer 1420 Multilabel Counter utilising the automated FITC parameter $(485 \mathrm{~nm} / 535 \mathrm{~nm})$.

\section{Cryo-sectioning of HydroMIPs (and HydroNIP controls)}

$\mathrm{BHb}$ and FITC-albumin HydroMIPs and non-imprinted polymers (NIPs) were prepared as detailed. $1 \mathrm{~cm}^{2}$ gel samples were cut with a clean razor blade, before being sectioned on a Lipshaw L500A Cryostat (Lipshaw, Pittsburgh, PA). The gel samples were mounted upon the cryostat chuck within M-1 embedding medium (Lipshaw, Pittsburgh, PA). The sample was allowed to freeze $\left(0^{\circ} \mathrm{C}\right)$ in the cryostat chamber for $10 \mathrm{mins}$, before mounting the chuck upon the cutting arm. Sections $(1 \mu \mathrm{m})$ were carefully cut and collected upon clean microscope slides, and stored in a humid chamber to ensure the gel sections did not dry out.

\section{Image Collection and Analysis of FITC-Albumin and BHb HydroMIPs}

All images of cryo-sectioned HydroMIPs and HydroNIP controls were collected using a Leica TCS SP2 AOBS spectral confocal microscope equipped with a Leica DMIRE 2 inverted microscope with three internal and two external photomultiplier tubes (PMT's). A MaiTai laser (Spectra Physics, 
Mountain View, CA) was incorporated by direct optical coupling, adding a twophoton excitation mode to the Leica confocal system. FITC-Albumin HydroMIP images were collected using a PL APO CS 63xHP 1.4 WD 100 $\mu \mathrm{m}$ oil immersion objective lens. All images were $512 \times 512$ pixels in size $(238 \mu \mathrm{m}$ $x 238 \mu \mathrm{m})$ and have a 12-bit pixel depth. The image analysis was performed using Adobe Photoshop. $\mathrm{BHb}$ autofluorescence images were collected using the MaiTai multiphoton laser source using the same objective as before. The sample was excited at $810 \mathrm{~nm}$ and the fluorescent emission was collected between 410-590nm. In all instances, control images of non-imprinted gels were obtained at identical experimental parameters to that of the respective HydroMIPs.

\section{BHb Autofluorescence Controls}

$12 \mathrm{mg} / \mathrm{ml}$ solution of $\mathrm{BHb}$ in $\mathrm{RO}$ water was made. A $100 \mu \mathrm{l}$ aliquot was pipetted onto a microscope slide and imaged using the MaiTai two-photon laser source using an oil source objective as before. The sample was excited at $810 \mathrm{~nm}$ and the fluorescent emission was collected between $410-590 \mathrm{~nm}$. Upon capturing the image, $100 \mu \mathrm{l}$ of a $10 \%(\mathrm{v} / \mathrm{v})$ acetic acid $(\mathrm{AcOH})$ solution containing $10 \%(\mathrm{w} / \mathrm{v})$ sodium dodecyl sulphate (SDS) was added to the $\mathrm{BHb}$ solution. An image was again captured in real time at identical experimental parameters. Control images of the $\mathrm{AcOH} / \mathrm{SDS}$ solution were also obtained.

\section{$\underline{\text { Results and Discussion }}$}

One of the key elements of any Ml process is the quantification of an "imprinting effect" exhibited by the HydroMIP, in relation to a HydroNIP control. Detection of protein in the wash and elution phases is indicative of 
certain MIP or NIP characteristics. The detection of protein in the "load" phase, indicates that protein has not bound within molecularly imprinted cavities in the gel. If protein is detected in the water only "wash" phase, it is likely that specific binding with molecularly imprinted cavities has not occurred. Evidence of protein in the "elution" phase (subsequent to "load" and "wash") is most indicative of an imprinting effect, as protein that has been selectively rebound within imprinted cavities will be eluted and detected in this phase. Detection of large amounts of protein in the elution phase (relative to the load and wash phases), suggests that a molecular imprint has been formed and conversely, elution of little protein suggests that no specific binding of protein occurred, and no molecular imprint was originally made. It is of great importance to display a significant retention effect of template by the MIP, when compared to that of the NIP.

Table 1 shows the distribution of recovered protein, having reloaded FITC-Albumin onto both a FITC-Albumin HydroMIP, and non-imprinted HydroNIP control. The NIP behaved in a typical manner, with $>75 \%$ of protein detected following the initial load, and the remainder in the subsequent water washes. No protein was detected in the elution phase, indicating that no, nonspecific binding occurred. The HydroMIP also behaved in a predicted manner, with significantly less FITC-Albumin being detected in the initial load or subsequent wash phases ( $16.8 \%$ and $25.9 \%$ respectively). The rebinding studies show that $57 \%$ of reloaded protein was detected in the elution phase. This strongly suggests that the reloaded FITC-Albumin bound within the molecularly imprinted cavities that had formed and subsequently been exposed following elution of the original template molecule. 


\begin{tabular}{|c|c|c|c|}
\hline & \multicolumn{3}{|c|}{ \% Distribution of Recovered Protein } \\
\hline & Protein Load & Wash & Elution \\
\hline MIP & 16.8 & 25.9 & 57.3 \\
\hline NIP & 77.5 & 22.5 & 0 \\
\hline
\end{tabular}

Table 1. Comparison of percentage distribution of recovered protein between a FITC-Albumin imprinted HydroMIP, and non-imprinted control. The "MIP" effect is characterised in terms of the observed percentage distribution of FITC-Albumin, following the reloading, washing and elution of the protein from the HydroMIP.

All confocal images were taken of the hydrogel based materials in their natural forms, with no physical manipulation of the gels being performed. Following polymerisation of the molecularly imprinted polymers, $1 \mu \mathrm{m}$ thin sections were cut upon a cryostat, thawed and then imaged. This minimalist approach to sample preparation not only allowed the imaging of the material in a native state, but allowed the real time in situ analysis of template removal events, as the structure had not been altered in a manner that would affect such phenomena.

Figure 1a shows a confocal micrograph projection image of an FITC labelled albumin imprinted HydroMIP. The highly crosslinked polyacrylamide structure is evident, with large aqueous voids interspacing the polymer matrix. The fluorescence that is evident relates to the FITC conjugates upon the imprinted albumin template, which have been entrapped within the polyacrylamide matrix during the free radical polymerisation procedure used to formulate the material. As approximately 8-12 FITC molecules are covalently conjugated to each albumin molecule, it is not appropriate to 
assume that each fluorescent molecule relates to a single protein specific cavity. However, the aggregation of fluorescent bodies is evidence of the existence of template protein embedded within the polyacrylamide material, occupying a molecularly imprinted cavity. Furthermore, the fact that fluorescence occurs within the hydrogel structure only and not the interspatial voids, further suggests that that the protein is entrapped solely within the polymer matrix. This supports the quantitative characterisation of Table 1 and strongly suggests that fluorescence is an indication of molecularly imprinted sites. Figure $1 \mathrm{~b}$ is an image of a non-imprinted polymer control taken at identical experimental parameters as Figure 1a. The distinct lack of any fluorescence indicates that the fluorescence observed in Figure 1a is due to the FITC conjugated protein and not autofluorescence exhibited by the polymer material.

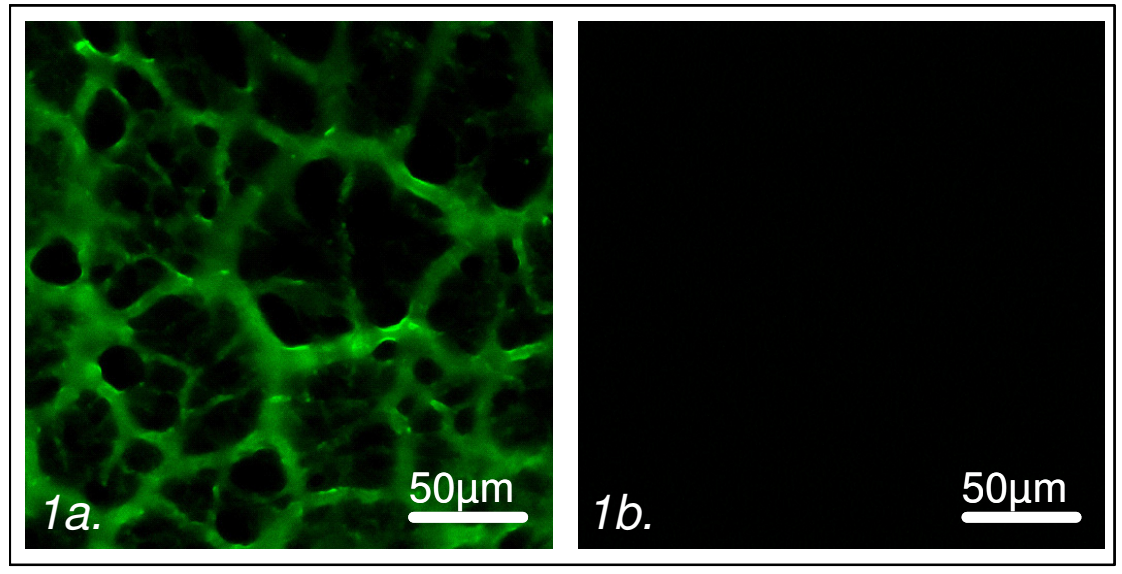

Figure 1. (a) A confocal intensity projection of an FITC-albumin imprinted HydroMIP image stack (18 optical sections) acquired with a z-step of $0.3 \mu \mathrm{m}$ and an image size of $238 \mu \mathrm{m} \times 238 \mu \mathrm{m}$. (b) A confocal HydroNIP control image acquired at the same conditions as Figure 1a. 
The molecularly imprinted materials that have been developed hold much promise for use in many different applications when employed as a specific and selective molecular recognition material. In order for a binding event to occur between a target template molecule and imprinted cavity, the original template molecule in which the polymer cavity complex was initially formed must first be removed. This template removal is a key element in any molecular imprinting protocol, and has been optimised in the aqueous phase as we have previously reported(3). SDS/AcOH has proven an excellent denaturation solvent for protein imprinting in the aqueous phase. Figure $2 a$ shows a confocal micrograph image of a FITC-albumin imprinted HydroMIP. As in Figure 1a, the fluorescent structure (which relates to the imprinted protein molecules) and polymer crosslinking is clearly evident, indicating the uniform and reproducible nature of the material. Figure $2 b$ is an image taken in the same focal plane, field of view, and experimental parameters as Figure $2 a$, following the addition of $50 \mu \mathrm{l}$ of $10 \% \mathrm{SDS} / 10 \% \mathrm{AcOH}(\mathrm{w} / \mathrm{v})$ denaturation solvent. An instant and almost total decrease in fluorescence was observed and occurred due to the structural denaturation of the template protein. 


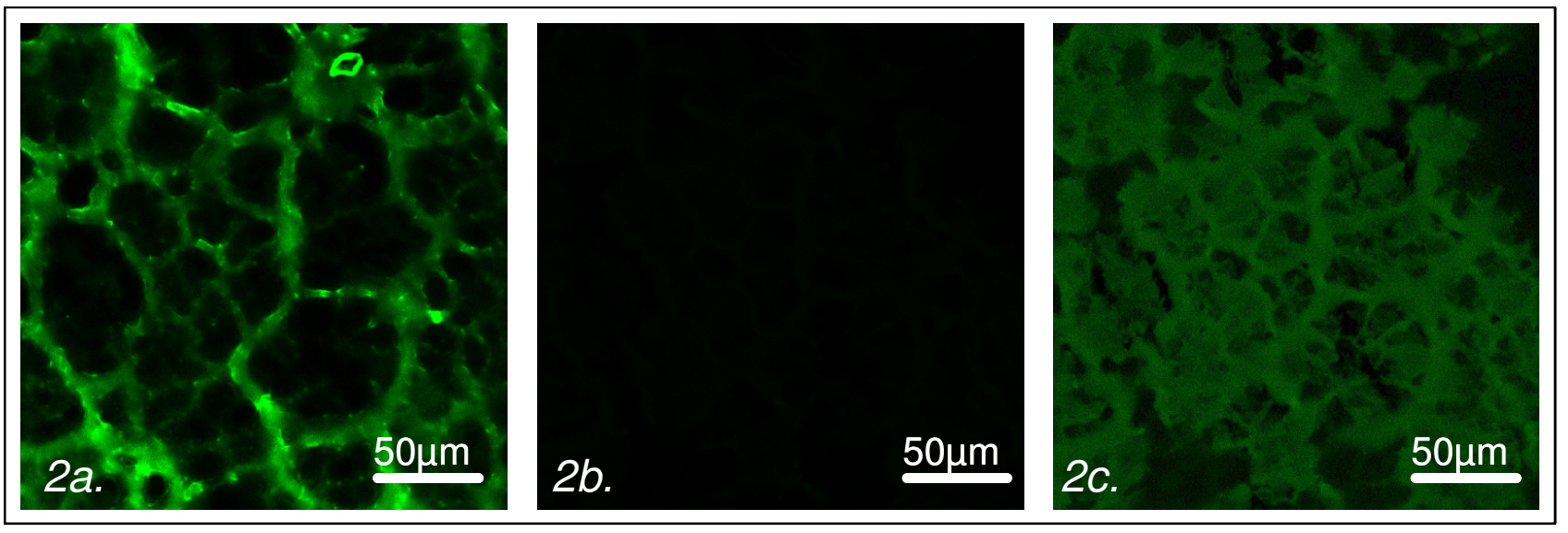

Figure 2. (a) A confocal image of an FITC-albumin imprinted HydroMIP. (b) A confocal image of an FITC-albumin imprinted HydroMIP (Figure 2a) acquired following the addition of $50 \mu \mathrm{l}$ of $10 \% \mathrm{SDS} / 10 \% \mathrm{AcOH}(\mathrm{w} / \mathrm{v})$. (c) $\mathrm{A}$ confocal image, identical to Figure $2 b$, having been optically enhanced. The maximum pixel intensity was $2.5: 2$, with a gamma value of $1: 2$. The profound decrease in fluorescence observed following the addition of the denaturation solvent was due to the removal of FITC-albumin from within the molecularly imprinted cavities of the polymer.

The unfolding of the template molecule was caused by the combinative chaotropic action of the acetic acid and the SDS surfactant solution (with the latter being above the critical micelle concentration (CMC)), which acted to unravel the globular protein structure and as a result, fluorescence decreased as the template molecule was removed from the imprinted polymer. Figure 2c is identical to that of Figure $2 \mathrm{~b}$, but has undergone post-capture optical enhancement using Adobe Photoshop. By enhancing the image intensity, it is possible to clearly identify the cross-linked structure of the hydrogel, which appears highly similar to that of Figure $2 b$, clearly demonstrating identical 
structural features distinguishable in both images. This is evidence alone that the gel network does not collapse, and that the decrease in fluorescence is a direct indication of template removal. The fluorescent template molecule has been removed to a great extent; however residual protein remains within the material and is responsible for the low level fluorescence exhibited. This suggests that not all of the template molecule has been removed. However, as detailed, the HydroMIPs imaged in this instance are in their natural form, and additional measures involving gel crushing and washing are taken to ensure maximal template removal occurs when the material is employed in a molecular imprinting protocol. Additionally, although the majority of polymer:template interactions are non-covalent in nature, a certain amount of covalent interactions also occur due to the free radical nature of the polymerisation reaction, resulting in a certain degree of irreversibly bound template molecule, which would contribute to the fluorescence observed in Figure 2c. Control images of non-imprinted polymers were taken at identical experimental parameters to those of figures $2 a, b \& c$ with no fluorescence evident whatsoever (not shown).

To date, much understanding and knowledge of aqueous phase molecular imprinting protocols has been gained through the investigation of the imprinting of haem containing protein molecules ${ }^{(1,2)}$. Haemoglobin is a relatively large globular serum protein, much like albumin, which plays a key physiological role in oxygen transportation, and is an important indicator of differing blood disorders. Although the need for novel sensing strategies for haemoglobin based conditions are not immediately apparent, the optimisation and understanding of imprinting protocols for molecules such as albumin and 
haemoglobin should aid understanding of how to optimise MIP synthesis for other clinically significant biological molecules ${ }^{(3)}$.

Unlike FITC labelled albumin, haemoglobin is not a highly fluorescent molecule. However, haemoglobin does exhibit a certain degree of natural fluorescence, or "autofluorescence" which can be imaged using an appropriate combination of filters. By definition, autofluorescence is the fluorescence of a molecule to which no chemical substances have been applied, and naturally evokes the emission of light at a higher wavelength to that of the light in which it was excited. The haemoglobin structure occurs as a result of the coordinated synthesis of a haem and globin group. Haem is synthesised following a series of complex mitochondrial enzymatic reactions, and following transportation to the cytosol, reaction occurs with a protoporphyrin IX molecule. Protoporphyrin IX is a tetrapyrolic pigment, which like all porphyrins exhibits natural autofluorescence, which in this application can be used to image the haemoglobin template molecule entrapped within the polymer matrix.

The haemoglobin HydroMIP was illuminated at 810nm using the MaiTai laser. Two-photon excitation of a fluorophore takes place when two photons are simultaneously absorbed by a fluorophore and result in the excitation of the fluorophore to a higher energy level that reflects the sum of the energy of the two arriving photons ${ }^{(16)}$.

Figure $3 \mathrm{a}$ is a confocal micrograph projection image of a $\mathrm{BHb}$ imprinted HydroMIP. Much like the corresponding FITC-albumin image (Figure 2a), a clearly defined crosslinked polyacrylamide structure is evident, upon which fluorescence relates directly to the template protein imprinted within. Again, 
fluorescence is specific to the polymer structure with no free protein evident within the interspatial voids. Figure $3 \mathrm{~b}$ is a confocal image taken at the same focal plane, field of view and experimental parameters as Figure $3 a$ following the addition of $50 \mu \mathrm{l}$ of $10 \% \mathrm{SDS} / 10 \% \mathrm{AcOH}(\mathrm{w} / \mathrm{v})$ denaturation solvent. An instant increase in autofluorescence is immediately observed. This differs greatly from the effect noted upon the addition of the denaturation solvent to the FITC-albumin HydroMIP where fluorescence decreased dramatically. This can be explained upon considering the highly folded quaternary structure of the protein ${ }^{(18)}$. Upon denaturation and unfolding of the structure, the molecule exposes an iron atom within the core of each of its four molecular subunits ${ }^{(19)}$. The iron, or haem group, is held in place by the naturally fluorescing heterocyclic porphyrin molecule that is quenched when the protein is in its highly folded globular form ${ }^{(20)}$. In effect, the denaturation and removal of the protein molecule from the HydroMIP structure increases autofluorescence due to the "unquenching" of the autofluorescing protoporphyrin IX complex. Further evidence to support this theory was obtained by conducting control experiments. 


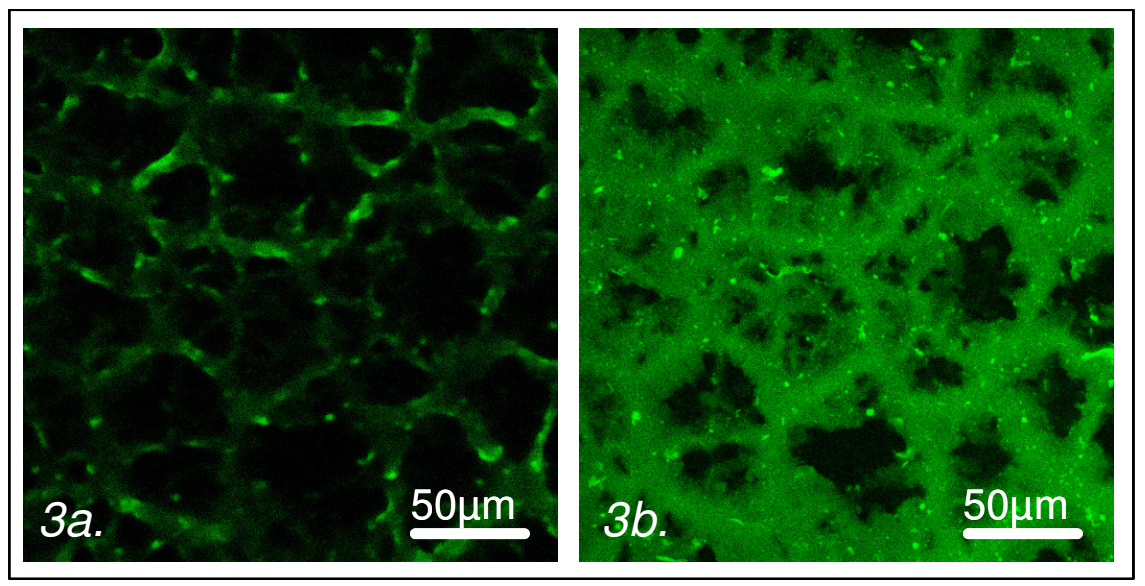

Figure 3. (a) A two-photon intensity projection of a $\mathrm{BHb}$ imprinted HydroMIP image stack (26 optical sections) acquired with a z-step of $0.3 \mu \mathrm{m}$. (b) A two-photon intensity projection of a BHb imprinted HydroMIP (Figure 3a) acquired following the addition of $50 \mu \mathrm{l}$ of $10 \% \mathrm{SDS} / 10 \% \mathrm{AcOH}(\mathrm{w} / \mathrm{v})$.

A $12 \mathrm{mg} / \mathrm{ml}$ haemoglobin solution was made and imaged using multiphoton confocal microscopy as previously described (Figure 4a). As expected, the resulting image shows a homogenously distributed autofluorescence, with localised regions of increased fluorescent intensity, which can be attributed to the incomplete dissolution of the concentrated protein in solution. Figure $4 \mathrm{~b}$ is an image taken at the same focal plane, field of view and experimental parameters as Figure 4a following the addition of $50 \mu \mathrm{l}$ of $10 \% \mathrm{SDS} / 10 \% \mathrm{AcOH}(\mathrm{w} / \mathrm{v})$ denaturation solvent. A marked increase in autofluorescence is observed that can be attributed to the unquenching of the porphyrin moieties as discussed. 


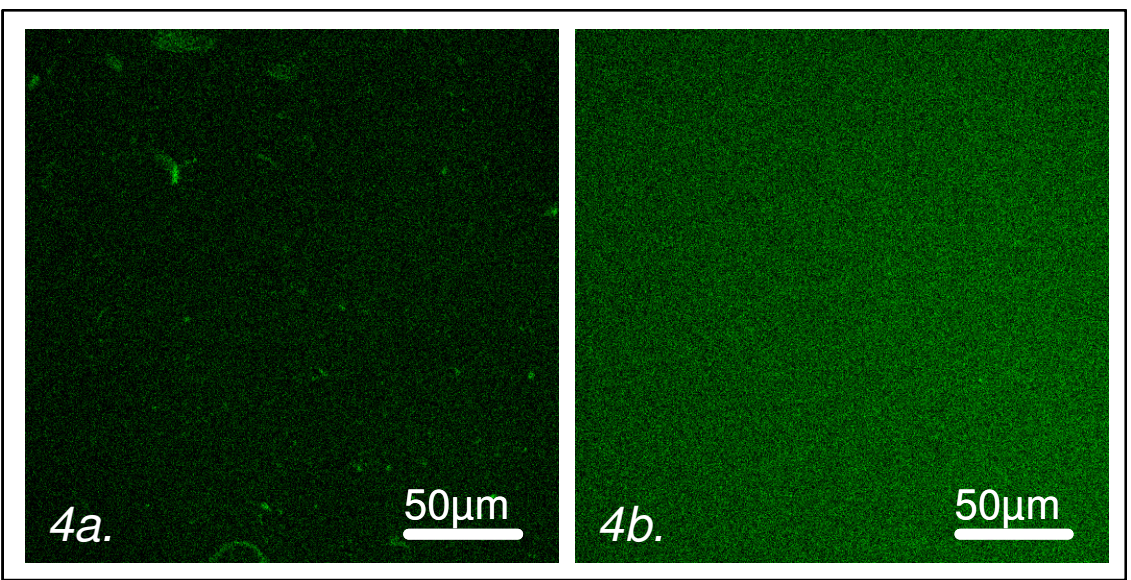

Figure 4. (a) A two-photon image of a $12 \mathrm{mg} / \mathrm{ml} \mathrm{BHb}$ solution (in $\mathrm{RO}$ water).

(b) A two-photon image of a $12 \mathrm{mg} / \mathrm{ml} \mathrm{BHb}$ solution (in $\mathrm{RO}$ water) following the addition of $50 \mu \mathrm{l}$ of $10 \% \mathrm{SDS} / 10 \% \mathrm{AcOH}(\mathrm{w} / \mathrm{v})$.

\section{Conclusions}

We report the design and development of an aqueous phase HydroMIP for a fluorescently labelled protein molecule of clinical significance. The quantification of the imprinting effect has been successfully performed and the selective recognition material has been imaged using confocal microscopy. The resulting information further adds to our understanding of aqueous phase molecular imprinting protocols, with the successful in situ imaging of real time protein denaturation events being of particular worth.

\section{Acknowledgements}

The authors would like to gratefully acknowledge the financial support provided by the Department of Trade and Industry, and the Engineering and Physical Sciences Research Council. Thanks also go to Dr. Shelagh 
Hampton of The University of Surrey for her assistance with the quantification of FITC-albumin imprinted polymers.

\section{References}

(1) Liao, J-L.; Wang, Y.; Hjerten, S. Chromatographia 1996, 42 (5/6), 359362.

(2) Ou, S.H.; Wu, M.C.; Chou, T.C.; Liu, C.C. Anal. Chim. Acta 2004, 504, 163-166.

(3) Hawkins, D.M.; Stevenson, D.; Reddy, S.M. Anal. Chim. Acta 2005, $542(1), 61-65$.

(4) Martin, P.D.; Wilson, P.D.; Wilson, I.D.; Jones, G.R. Analyst 1999, $126(6), 757-759$.

(5) Gao, S.; Wang, W.; Wang, B. Bio-org. Chem. 2001, 29, 308320.

(6) Tong, A.; Dong, H.; Li, L. Anal. Chim. Acta 2002, 466, 31-37.

(7) Wandelt, B.; Mielniczak, A.; Turkewitsch, P.; Wysocki, S. J. of Luminescence 2003, 102-103, 774-781.

(8) Rathbone, D.L.; Ali, A.; Antonaki, P.; Cheek, S. Biosens and Bioelec. 2005, 20 (11), 2353-2363.

(9) Shinkai, S.; Takeuchi, M. Biosens. and Bioelec. 2004, 20 (6), 12501259.

(10) Lulka, M.F.; lqbal, S.S.; Chambers, J.P.; Valdes, E.R.; Thompson, R.G.; Goode, M.T.; Valdes, J.J. Mat. Sci. and Eng. C 2000, 11, 101105. 
(11) Suarez-Rodriguez, J.L.; Diaz-Garcia, M.A. Anal. Chim. Acta 2000, 405, 67-76.

(12) Charles, P.T.; Goldman, E.R.; Rangasammy, J.G.; Schauer, C.L.; Chen, M.S.; Taitt, C.R. Biosens. and Bioelec.. 2004, 20, 753764.

(13) Gunnlaugsson, T.; McCoy, C.P.; Stomeo, F. Tetrahed. Lett. 2004, 45, 8403-8407.

(14) Crompton, K.E.; Prankerd, R.J.; Paganin, D.M.; Scott, T.F.; Home, M.K.; Finkelstein, D.I.; Gross, K.A.; Forsythe, J.S. Biophys. Chem. 2005, 117, 47-53.

(15) Geustens, G.; Soukrati, A. Eur. Poly. J. 2000, 1537- 1546.

(16) Matsumura, Y.; Iwai, K. Polymer 2005, 46, 10027-10034.

(17) Hibbs, A.R. Confocal Microscopy for Biologists, $1^{\text {st }}$ ed.; Eds.; Kluwer Acedemic/Plenum Publishers: New York, 2004; Chapter 3, pg 94.

(18) Silva, M.M.; Rogers, P.H.; Arnone, A. J. of Bio. Chem. 1992, 267, 17248-17256.

(19) Perutz, M.F.; Rossman, M.G.; Cullis, A.F.; Muirhead, H.; Will, G.; North, A.C.T. Nature 1960, 185 (4711), 416-422.

(20) Kuszaj, S.; Kaszycki, P.; Wasylewski, Z. Chem. and Phys. of Lipids 1996, 83, 153-160. 


\section{For Table of Contents Use Only}

“The Quantification and Confocal Imaging of Protein Specific

Molecularly Imprinted Polymers (HydroMIPs)"

Daniel M. Hawkins, Andreea Trache, E. Ann Ellis, Derek Stevenson, Andreas

Holzenburg, Gerald A. Meininger, \& Subrayal M. Reddy.

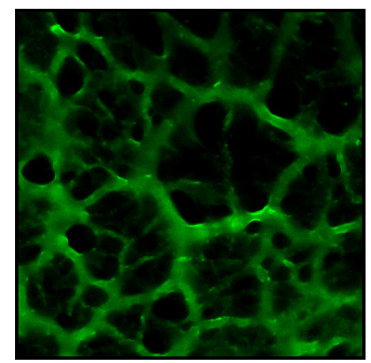

